\title{
LA REPRODUCCIÓN DE LAS DESIGUALDADES DE GÉNERO EN CONTEXTOS MIGRATORIOS Y LABORALES. EL CASO DE MIGRANTES BOLIVIANOS Y BOLIVIANAS EN TORNO AL MERCADO DE TRABAJO HORTÍCOLA DE SALTA (ARGENTINA)
}

\author{
Reproduction of gender inequalities within migratory and labor \\ contexts. The case of male and female migrants in the \\ horticultural labor market of Salta (Argentina)
}

Soraya Ataide*

\begin{abstract}
Resumen. El siguiente artículo presenta los hallazgos de una investigación, que tuvo como objetivo indagar en la reproducción de las desigualdades de género presentes en un contexto migratorio y laboral. Particularmente en migrantes de origen boliviano, unidos por relaciones de pareja quienes en destino (la provincia de Salta, en el Noroeste argentino) se articularon como trabajadores en la producción hortícola. Con dicho objetivo optamos por un enfoque metodológico cualitativo y siguiendo una estrategia etnográfica.
\end{abstract}

Palabras clave: género; migraciones; bolivianos y bolivianas; mercado de trabajo hortícola; Salta (Argentina).

\begin{abstract}
This work introduces the findings of a research that was aimed at inquiring into reproduction of gender inequalities within a specific migratory and labor context. Particularly, the research focused on Bolivian migrant couples who became male and female horticultural workers once in destination (in the Province of Salta, in the northwest of Argentina). According to this research objective, a qualitative methodology was carried out and an ethnographic strategy was used.
\end{abstract}

Keywords: gender; migrations; male and female Bolivians; horticultural labor market; Salta (Argentina).

Consejo Nacional de Investigaciones Científicas y Técnicas (CONICET), Universidad Nacional de Salta, docente de la escuela de Agronomía de la FCN de la Universidad Nacional de Salta. San Lorenzo, Salta, Argentina. E-mail: soraya.ataide@gmail.com. Orcid: 0000-0003-4479-5012. 


\section{Introducción}

El noroeste argentino ${ }^{1}$-NOA- constituye un destino temprano para la migración boliviana, inclusive el fenómeno antecede la conformación de ambos estados (argentino y boliviano, respectivamente). Hasta las primeras décadas del siglo XX esta migración se vinculó como mano de obra barata en las principales actividades desarrolladas en dicha región: la caña de azúcar y el tabaco. No obstante, a mediados de siglo, con la crisis en ambas producciones, el AMBA (Área Metropolitana de Buenos Aires) se convirtió en su primer destino. Y desde entonces se reconoce una importante expansión de esta migración en distintos puntos del territorio nacional (Giarraca, 2003), tanto en ámbitos rurales como urbanos.

Asimismo, el NOA mantiene su importancia, conformando la segunda región elegida en Argentina, por bolivianos y bolivianas quienes, desde la década de 1990 comenzaron a insertarse fuertemente en la actividad hortícola, orientada al consumo en fresco y con destino al mercado interno. Esto se observa en distintos lugares de Salta (Km 28, Colonia Santa Rosa, Gral. Güemes, Apolinario Saravia, Gral. Pizarro y el cinturón verde de la ciudad de Salta), en Jujuy (Perico) y Tucumán (Lules) entre otros lugares. No obstante, la vinculación de bolivianos/as en esta actividad, no es exclusiva del NOA más bien constituye un fenómeno presente en distintas zonas del país, por lo menos desde finales de la década de 1980.

En relación a esta temática existen abundantes investigaciones ${ }^{2}$, principalmente enfocadas en los lugares de destino de los/as migrantes y en particular en los espacios de trabajo. Estudios que dan cuenta de las precarias condiciones de vida y trabajo de estos/as migrantes, a quienes además se les asignan ciertas características como innatas por su condición nacional, favorables para el trabajo duro y sacrificado como es el que caracteriza al trabajo en la horticultura (Pizarro, 2013). Básicamente se construyen discursos que naturalizan y justifican la inserción laboral segmentada por nacionalidad boliviana en ese nicho laboral.

Por otra parte, se reconocen las estrategias que ponen en juego estos migrantes para -en ciertos casos- lograr una movilidad social ascendente (Benencia, 2012). Lo cual se reconoce en quienes inician sus trayectorias laborales como peones o mensualeros ${ }^{3}$, luego se insertan como medieros ${ }^{4}$, a

1 Las regionalizaciones suelen incorporar en el NOA (Noroeste argentino) a las provincias de Jujuy, Salta y Tucumán. En algunos casos también a Santiago del Estero, no obstante, la migración boliviana ha sido particularmente fuerte en las tres primeras.

2 Podemos mencionar los trabajos de Benencia (2012); García (2012) y Pizarro (2011 y 2012) entre otros.

$3 \mathrm{El} / \mathrm{la}$ mensualero/a refiere al trabajador/a que recibe un salario fijo acordado previamente con el patrón o dueño de las tierras.

4 En la mediería hortícola de la zona estudiada (Gral. Pizarro y Apolinario Saravia en la provincia 
veces logran convertirse en productores arrendando tierras o adquiriéndolas vía compra, mientras que en otros casos inclusive llegan a desempeñarse en el eslabón de la comercialización.

Con respecto al mercado laboral hortícola, este se encuentra segmentado fuertemente por género, además de por nacionalidad. Con esto queremos significar el hecho de que, si bien el trabajo en la horticultura suele ser desarrollado por varones y mujeres (muchas veces parejas de migrantes bolivianos), los arreglos laborales y comerciales son frecuentemente realizados entre varones; además, en la sociedad de destino, el trabajo en la horticultura es considerado como un trabajo para varones. Podemos inferir que estas cuestiones tienden a invisibilizar el rol de las mujeres bolivianas en la horticultura. Y esta invisibilización se acentúa a su vez porque sus trayectorias migratorias no son autónomas sino que responden, más bien, al tipo de reunificación familiar, por arrastre o asociacional (Ataide, 2017). Todo esto permite comprender por qué su participación en este nicho laboral ha sido poco estudiada hasta el momento.

Precisamente, analizar la dimensión de género resulta novedoso puesto que los estudios sobre la articulación de bolivianos en el mercado laboral hortícola se suelen centrar más en la dimensión de nacionalidad de los trabajadores que en la dimensión de género. En ese contexto, pretendemos aportar a los estudios que vinculan género y migraciones, con un estudio de caso que focaliza en la movilidad de bolivianos y bolivianas de origen campesino, procedentes de distintas zonas rurales de Tarija con destino al NOA y en particular en torno a la producción hortícola de la provincia de Salta. La investigación tuvo como objetivo indagar en la reproducción de las desigualdades de género, en varones y mujeres migrantes unidos por relaciones de pareja quienes se articularon como trabajadores, generalmente, bajo la figura de la mediería en la producción hortícola.

La mediería en la horticultura constituye un arreglo laboral, donde el patrón aporta no sólo la tierra sino también todos los insumos necesarios para desarrollar el ciclo productivo, inclusive decide qué se produce, cómo y cuánto; mientras la pareja de migrantes, únicamente aporta su fuerza de trabajo. Ciertamente, poco se ha investigado sobre las relaciones desiguales de poder y sus consecuencias, al interior de estas parejas de migrantes que trabajan juntos, para lo cual es necesario considerar las particularidades del sistema de género en origen y destino, como también del mercado de trabajo donde se insertan.

de Salta) el trabajador o los trabajadores suelen acordar con el patrón o dueño una remuneración por el trabajo que corresponde a un porcentaje de la venta de la producción que suele variar entre un 30 y un $35 \%$ de la misma. 
En nuestro análisis de la unidad doméstica conformada por parejas de origen tarijeño y campesino, establecidos en Apolinario Saravia (provincia de Salta) hemos observado una tendencia hacia la reproducción de la filosofía patriarcal de origen. Donde es el varón quien detenta el rol de autoridad en la esfera laboral/productiva/pública y también doméstica. Por su parte, esta continuidad de los roles y relaciones de género de origen, a partir de la migración no entraron en contradicción con el sistema de género en destino. Dicho sistema de género también coloca a los varones en las jerarquías decisorias del ámbito público y doméstico.

La afirmación precedente se sustenta en tres cuestiones que surgieron de la investigación. i) En primer lugar, a partir del rol predominante de los varones en los espacios de capacitación o discusión de políticas en torno a la actividad hortícola en Salta; como también en los arreglos laborales y comerciales. Y la ausencia de las mujeres en los mismos. ii) A su vez, hemos reconocido que estos espacios de toma de decisiones en la esfera productiva estaban cooptados por varones en los lugares de origen en Tarija. Por otro lado, iii) las limitaciones que mencionaron las mujeres para articularse como trabajadoras en la horticultura y por consiguiente las condicionalidades que surgen en las relaciones conyugales en presencia de conflictos.

No obstante esto, pudimos visualizar ciertas condiciones de contexto en destino que permitieron contrarrestar las situaciones de opresión o subordinación familiar y laboral de estas mujeres. En primer lugar, la construcción de redes de contención femenina, vinculadas a la antigüedad de la migración. Por otro lado, condiciones relacionadas a la implementación de ciertas políticas públicas desde finales de la década del 2000, como la implementación de la tarjeta social ${ }^{5}$ y, principalmente, la aprobación de la Asignación Universal por Hijo -AUH ${ }^{6}$.

En nuestra investigación, concebimos al género como un conjunto de prácticas, símbolos, representaciones, normas y valores sociales que las sociedades elaboran a partir de la diferencia sexual anátomo-fisiológica (De Barbieri, 1992). Así, se construyen formas hegemónicas de ser femeninos y masculinos como también se moldean los modos en que los géneros se relacionan y, estas relaciones están generalmente atravesadas por desigualdades en perjuicio de las mujeres.

5 Esta tarjeta es gestionada por un/a jefe/a de hogar, en situación de vulnerabilidad, de forma gratuita en la oficina de Acción Social de cada municipio. Corresponde a un ingreso económico a partir de \$ 70 (hasta el año 2015) según la cantidad de hijos del grupo familiar, destinado a la compra de alimentos.

6 La AUH es un beneficio que le corresponde a los hijos de las personas desocupadas, que trabajan en el mercado informal o que ganan menos del salario mínimo, vital y móvil. Consiste en un pago mensual en pesos para niños menores de 18 años y para personas con discapacidad sin límite de edad. La misma fue implementada en el año 2009 y es otorgada a las madres o tutoras. 
Los hallazgos que presentamos en el artículo están referidos a un proceso de investigación con enfoque etnográfico, basado en entrevistas en profundidad y observación participante en Apolinario Saravia (provincia de Salta) uno de los lugares de destino de estos/as migrantes y también en Iscayachi, Tolomosa Grande y San Jacinto (departamento de Tarija, en Bolivia) correspondientes a los lugares de origen de los/as mismos/as. Realizamos un total de 33 entrevistas que corresponden a 14 varones y 19 mujeres de origen boliviano, quienes iniciaron sus trayectorias migratorias en distintos momentos, entre 1980 y la década del 2000.

En su mayoría, los varones iniciaron la migración previo a que lo hicieran las mujeres, quienes luego migraron junto con ellos, representando una típica migración de arrastre o por reunificación familiar. En menor medida (sólo tres casos) identificamos migraciones femeninas autónomas. En esos casos, las mujeres migraron hacia Argentina a través de redes constituidas por varones que integraban su familia nuclear o ampliada y una vez en destino, conformaron parejas con varones de sus lugares de origen, con quienes se asociaron para trabajar en la horticultura.

\section{Algunos antecedentes para pensar en la vinculación entre género y migraciones}

La producción científica sobre migraciones ha estado marcada históricamente por su carácter economicista y androcéntrico (Herrera, 2012), desde la misma se ocultó el papel de las mujeres migrantes debido a la suposición ampliamente compartida de que ellas (y los/as niños/as) migraban para acompañar o para reunirse con los esposos/padres (Mahler, Pessar, 2006). Recién hacia la década de 1970, investigadoras feministas incorporaron la dimensión de género en los estudios migratorios, aunque con una mirada puesta casi exclusivamente en las mujeres. Luego, dentro de los estudios postestructuralistas se propuso un abordaje de los sistemas de género más relacional y situacional.

Hacia la década de 1980 se incorporó el enfoque interseccional buscando analizar cómo operan las desigualdades de raza, clase y género, poniendo en discusión la categoría de mujer universal, lo cual enriqueció el análisis - particularmente- de las mujeres migrantes. Asimismo, se rechazó la idea de la familia como espacio librado de relaciones de poder y se planteó la existencia del género como estructurador de las relaciones en su interior (Mahler, Pessar, 2006), visibilizando el sistema patriarcal y la dominación masculina. Avanzada la década de 1990, comenzó a hablarse de feminización cuantitativa y cualitativa de las migraciones. La primera tenía como principal objetivo visibilizar la creciente presencia de mujeres migrantes en ciertos flujos 
migratorios. Mientras que desde la feminización cualitativa se plantearon críticas a la visión androcéntrica del fenómeno.

De acuerdo con Ariza (2007), actualmente asistimos a una nueva fase en los estudios sobre género y migraciones que supera a aquella que se circunscribía a las relaciones intra familiares. Según la autora existe un intento de analizar cómo el género atraviesa las diversas prácticas, identidades e instituciones que intervienen en el proceso migratorio. En definitiva, existe un acuerdo en concebir al sistema de género como estructurador de las migraciones y en que la migración es uno de los factores que con mayor fuerza alteran y realinean la vida diaria de las personas que migran.

Dentro de este campo de estudios uno de los principales interrogantes giró sobre los procesos de autonomía en las mujeres y de la posible mejora en la condición de opresión o subordinación con respecto a los varones, como consecuencia de la migración. Sin embargo, diversas investigaciones reflejan la reproducción de la desigualdad en las mujeres migrantes (Gregorio Gil, 1998; Stefoni, 2004; Magliano, 2009; Courtis, Pacceca, 2010; Bastia, 2014). En ese sentido, Pizarro (2015) reconoce que si bien en algunos casos, la migración puede resultar en nuevas oportunidades para cruzar las fronteras de género, la operatoria de otro tipo de fronteras y jerarquías sociales puede generar nuevas formas de dependencia dentro de las familias y en otros contextos más amplios. Esto se debe, en primer lugar, a que el ámbito donde transcurren las migraciones es la unidad doméstica, la que está atravesada por relaciones de poder; en segundo lugar, porque los mercados de trabajo donde se insertan no sólo están generizados sino que también están segmentados por etnia, nacionalidad, clase y condición migratoria (Pizarro, 2013).

Entonces, el grado de autonomía que logran las mujeres migrantes y su vínculo con respecto a los varones virará de acuerdo a la manera en que puedan transformar, reproducir o reforzar los modelos de género establecidos, la dependencia familiar, la inserción subalterna en mercados de trabajo segmentados y las discriminaciones étnico-nacionales (Pizarro, 2013). Es decir, la transformación en las relaciones de poder entre varones y mujeres y la posibilidad de romper con mandatos de género constituye algo a investigar y no debe ser dado por supuesto. Veamos.

\section{Las formas en que se reproduce la desigualdad en contextos migratorios y laborales}

En la mayoría de las entrevistas tanto en varones como en mujeres, uno los principales factores que aparecieron explicando la migración fue la limitación en la reproducción social de las unidades domésticas campesinas 
en los lugares de origen. Especialmente, como consecuencia de la escasez de agua, de tierras y de circulación de dinero. Asimismo, observamos que los sistemas productivos campesinos se caracterizaban por la producción de bienes de uso, generalmente destinados al auto consumo. Entonces, en una economía campesina limitada para reproducir a sus integrantes, las unidades domésticas adoptaron como estrategia de reproducción socio-económica, la migración de los mismos. A su vez, esta migración fue impulsada a través de la memoria colectiva (Cassanello, 2014), trasmitida a través de relatos de experiencias de otros/as migrantes, favoreciendo la construcción de una imagen del lugar de destino como atractivo por la posibilidad de acceder a un trabajo y dinero para adquirir bienes impensados en el contexto de origen.

En el caso de los varones, el contexto de origen adverso se articuló con ciertos mandatos masculinos que permiten explicar la migración a ciertas edades y bajo modalidades particulares. Entre los catorce y los veinte años aproximadamente es el momento en que los varones se "hacen jóvenes", esto significa un cambio de etapa en el ciclo vital. Los varones dejan de ser niños, entonces tienen que valerse por sí mismos, "salir a buscar", y eso significa ir donde se puede encontrar trabajo, lo cual tiene entre las opciones la migración.

Otra de las cuestiones que surgió de las entrevistas es que, en su mayoría, los varones comenzaron sus trayectorias migratorias siendo solteros, en estos casos la migración no se explicaba en el mandato de sostener a sus familias, como proveedores, pero sí sostenerse a ellos mismos en términos económicos y, como consecuencia, aliviar la carga familiar. Es decir, de alguna manera la migración es siempre colaborativa de la supervivencia familiar (Mallimacci Barral, 2012). Ahora bien, en algunos casos, estos varones, luego de varios años de migrar de forma estacional (ir y venir) inician un nuevo proyecto migratorio junto con la pareja heterosexual constituida en origen.

Este nuevo proyecto migratorio es diferente al anterior porque tiene como objetivo trabajar en la agricultura junto a la mujer con quien conformaron una relación de pareja y establecerse definitivamente en destino. Entonces podemos reconocer que la migración de esas mujeres bolivianas, no se corresponde con el diagnóstico de la feminización de las migraciones. Además encontramos pocas mujeres que iniciaron su migración de forma autónoma, o más bien sin una pareja. Por eso, consideramos que en general, la movilidad territorial de estas mujeres se enmarca en una migración de arrastre o por reunificación familiar. No obstante esto, no significa que su rol en la esfera productiva y laboral sea irrelevante, más bien se encuentra invisibilizada y fuertemente subordinada a las decisiones de los varones. Veamos. 
María Ester ${ }^{7}$ (sesenta años, nacida en Iscayachi) comienza su migración a los veintidós años junto con Carlos, su marido, quien ya tenía experiencia migratoria previa hacia Argentina:

...años antes no había ninguna ayuda para criar a los niños, yo veía que se morían muchos chicos por lo que no tenían remedios, falta de atención, vacunas. Yo decía a mi esposo, yo no quiero tener los hijos aquí, no tenemos ninguna ayuda. Cuando helaba no había plata y nosotros, una pareja que quería tener familia, no había nada... ${ }^{8}$

Otro caso es el de Natalia, nacida en Tolomosa Grande y que al momento de la entrevista tenía cuarenta años y llevaba más de veinte en la Argentina. Al igual que María Ester migra con un proyecto de pareja, reconociendo que su decisión estuvo asociada a "seguir los pasos de su marido"9.

Por su parte, María (de treinta y ocho años, nacida en Iscayachi) migra para reunirse con Raúl quien había iniciado su experiencia migratoria siendo soltero. Raúl, "iba y venía cada año. Y traía dinero"10, ese bien que era prácticamente desconocido en las unidades domésticas campesinas de Iscayachi. El dinero para María significaba la posibilidad de adquirir ciertos bienes que desde su economía campesina no podía acceder. Y ciertamente, era el varón quien cumplía el rol de proveedor saliendo a migrar-trabajar. Sin embargo, María también contó que era un "poco irresponsable", porque "dejan con hijos y se van"11. Dando cuenta de que en ciertos momentos la migración de Raúl no significaba la posibilidad de proveerse de lo necesario para subsistir.

Años después, María migra con sus tres hijitas pequeñas para reunirse con Raúl en Salta. Así lo cuenta: "yo también medio obligada a venir, porque, qué iba a hacer con tres chicas. Y allá era feo, esos años eran feos, para comer a veces faltaba, mucho sufría de hambre"12. De esa manera reconocemos que en ciertas ocasiones la migración del varón puede significar un aumento del empobrecimiento de las mujeres, como también una multiplicación en las cargas de trabajo. María se sentía obligada a migrar por su situación económica y principalmente por la falta de posibilidades de mantener a sus hijas. Esto contrarrestaba la idea de la migración por amor o vehiculizada por relaciones conyugales. Esta mujer veía en la reunificación con el padre de sus hijas una posibilidad de subsistencia para ella y las niñas. Entonces, nuevamente, apareció el rol de madre/cuidadora como móvil de la migración.

\footnotetext{
7 A lo largo del artículo se utilizan seudónimos para resguardar la identidad de los y las entrevistados/as.

\& Comunicación con María Ester, Apolinario Saravia, provincia de Salta, mayo de 2016.

9 Comunicación con Natalia, Apolinario Saravia, provincia de Salta, septiembre de 2015.

${ }^{10}$ Comunicación con María, Apolinario Saravia, septiembre de 2015.

11 Comunicación con María, Apolinario Saravia, septiembre de 2015.

12 Comunicación con María, Apolinario Saravia, septiembre de 2015.
} 
En definitiva, si bien el contexto en el cual se enmarca la migración de las mujeres es similar al de los varones, ellas construyen un proyecto migratorio de modo diferente. En primer lugar, estas mujeres tuvieron que negociar el permiso de migrar con los varones de su unidad doméstica, padres o hermanos mayores. Y la autorización de hacerlo estuvo vinculada al hecho de migrar con un varón, para constituir sus propias unidades domésticas en destino, en tanto ámbito de realización de la maternidad (Ariza, 2007). Precisamente esta es la forma legítima de migrar para estas mujeres. Asimismo, esa migración significó trabajar junto con los varones y en ese sentido parte de lo que motoriza la migración es la posibilidad de insertarse en un mercado laboral. Estas mujeres se articularon rápidamente en la producción hortícola, pero como veremos, en condiciones de mayor vulnerabilidad que sus pares varones.

Estas mujeres se insertaron en un mercado laboral segmentado por género. La producción hortícola constituye una actividad donde las decisiones técnicas y los arreglos laborales y comerciales suelen realizarse entre varones. Durante nuestro trabajo de campo en Apolinario Saravia (Salta), presenciamos tres capacitaciones y varias reuniones de productores, del sector hortícola convocadas, organizadas o en presencia de organismos del Estado Nacional y Provincial. En las mismas observamos la presencia únicamente de varones. Entendemos que esto se vincula al hecho de que dentro de la actividad, se espera que sean los varones quienes estén involucrados en las decisiones técnicas y organizativas vinculadas al manejo de la finca.

Ahora bien, esta jerarquía masculina también se reconoce en origen. Podemos mencionar dos situaciones que ocurrieron en Iscayachi. La primera sucedió en febrero de 2016, dialogando con una familia campesina de la zona preguntamos si podíamos ver los cultivos que ellos realizaban y, en ese momento, estando presentes la mujer, su esposo, un hijo y un nieto, fue el padre, el varón mayor de la familia quien nos condujo por la recorrida. En su rol de representante de la unidad doméstica nos contó sobre los cultivos, las formas de riego y las problemáticas productivas. La mujer permaneció en el hogar cocinando. El otro suceso ocurrió en febrero de 2017 cuando visitamos otra familia en Iscayachi. Entonces el varón no estaba presente en el hogar porque se encontraba reunido con otros campesinos de la zona para acordar acciones productivas en los campos. Podríamos inferir que, al igual que aquel espacio de discusión de problemáticas y posibles líneas de acción política existentes en Apolinario Saravia (Salta), también en el campesinado tarijeño, esos espacios de encuentro entre productores tiene a los varones en un rol protagónico.

Por otra parte, nos referiremos a las condiciones de vulnerabilidad de las mujeres bolivianas en la esfera laboral y su vinculación con la subordinación que vivencian en la esfera doméstica. Nos interesa plantear, en primer lugar, 
el modo en que la etapa del ciclo vital que coincide con la maternidad, incide fuertemente en las mujeres limitando su accionar. Juana relató el momento en que nace uno de sus hijos y tuvo que ser internada en otra localidad. Comentó que al permanecer internada en el hospital sentía que dejaba solos/as a sus otros/as hijos/as. Lo extraño es que no mencionó el hecho de que el resto de sus hijos/as se encontraban viviendo también con el padre. Esto da cuenta de una concepción de la paternidad que aleja a los varones de las tareas de cuidado de los/as hijos/as. Ella recién se tranquiliza cuando su madre viene desde Tarija a cuidar de ellos/as.

J: Si, al principio sí te sentís sola, después ya no. Yo tuve eso así, cuando me han hecho la cesárea de Axel, yo vivía en la finca, de ahí me derivaron a González ${ }^{13}$. Yo ya tenía a Brisa, Érica y Esteban [...] en ese momento pensaba, y si yo no vuelvo mis hijos con quien van a quedar, era una desesperación. Después hablé a Bolivia, allá me contestó mi hermano, que me quede tranquila que dentro de dos, tres días ya iba a venir la mami [...] Es una angustia grande que te agarra, y bueno, después ya vino la mami, ya pasó, se quedó como quince días. ${ }^{14}$

Luego, esta mujer compara aquel momento pasado, cuando sus hijos/ as eran muy pequeños/as, con su presente donde ella se encuentra más liberada de las tareas domésticas, porque es reemplazada en parte por sus hijas, quienes reproducen ese rol feminizado. $Y$ esas tareas que forman parte del trabajo doméstico no son concebidas como "trabajo" sino como ayuda.

S: ¿Cómo se hace con el doble trabajo, en la finca y en la casa?

J: Tiene que volver y seguir haciendo en la casa. iAy! iQué pesado que era antes! iUna pesadilla, como para no acordarse! Ahora por ejemplo, tengo mis nenas más grandes, una va a la mañana al colegio y la otra va a la tarde. Ella, la que se queda a la mañana ya cocina, eso es una ayuda muy grande... ${ }^{15}$

Cuando la conocimos, Juana se encontraba en crisis con su pareja y sobre la posibilidad de separarse manifestaba: “ies muy difícil separarse! ¿Qué voy a hacer yo? ¿De qué voy a vivir? Nadie me va a querer dar trabajo"16. Ciertamente, estaba manifestando que en la producción hortícola, una mujer sola no podría realizar un arreglo contractual de trabajo con un patrón. De ese modo, entendía que su condición de género la condicionaba en su inserción laboral y en definitiva en su capacidad de sostener económicamente a la unidad doméstica.

\footnotetext{
${ }^{13}$ Se refiere a Joaquín V. González, ciudad cabecera del departamento de Anta.

${ }^{14}$ Comunicación con Juana, Apolinario Saravia, octubre de 2016.

${ }^{15}$ Comunicación con Juana, Apolinario Saravia, octubre de 2016.

${ }^{16}$ Comunicación con Juana, Apolinario Saravia, septiembre de 2015.
} 
Aquella entrevista con Juana, transcurría mientras su marido negociaba con los compradores el precio de los melones. Como mencionamos, los arreglos comerciales en la actividad son realizados entre varones, en su rol vinculado al espacio público, reproduciendo la filosofía patriarcal. Pero también quisiéramos plantear otra situación que nos permitió inferir las relaciones de poder que se juegan al interior de la pareja y en vinculación con la organización del trabajo. En cierta ocasión, por su gesto de cansancio le pregunté por el trabajo y dijo:

No, cansancio no, amargura. Hoy me enojé tanto, me quise venir de la finca, yo me voy a trabajar con él para que él salga con el hermano y se vaya a tomar, se gasta el dinero [...] Yo a él, hace poco vino, me quiso pegar yo no dude y fui a la policía, así como estaba, descalza [...] Me dio bronca, porque él vino y me hizo pasar vergüenza, en toda la gente que vive alrededor, toda la gente escucha, y agarré al menor de mis hijos y me fui. ${ }^{17}$

¿Qué sucede cuando hay un conflicto en la pareja? ¿Quién tiene mayor autonomía para disponer del tiempo o el dinero? De acuerdo con el relato es el varón quien tiene el poder para irse cuando quiera y dejarla trabajando. Juana relató dos formas de violencia, una psicológica porque su marido "se gasta la plata" que es ganada a partir del trabajo de ambos. Y en ese acto de gastarse la plata sin el acuerdo de Juana está demostrando que tiene el derecho de hacerlo, que puede disponer de ese dinero sin su consentimiento. Inclusive, la violencia psicológica está presente porque ella sufre lo que escuchan sus vecinos. También apareció la violencia física: "me quiso pegar". Ambas son disciplinadoras del sistema de género donde el varón es quien alecciona, reflejando relaciones de poder desigual.

Con respecto a la denuncia policial que realiza Juana por la violencia que sufrió por parte de su marido nos permitió hacer un análisis sobre los marcos de contención que favorecieron mayores niveles de autonomía en las mujeres. La presencia de una red de contención (amigas, vecinas, comadres) seguramente ya existía en la vida de Juana. Lo que queremos resaltar es que evidentemente los años transcurridos en destino y los vínculos con otras mujeres, le permitieron a Juana accionar sobre la situación y hacer la denuncia. Una denuncia quizás impensada en los primeros tiempos de la migración.

Veamos ahora el caso de Natalia, quien también migra con su pareja y se inserta junto a él como mediera en la producción hortícola en la ciudad de La Plata ${ }^{18}$. Lo particular en su trayectoria, que no hemos visto en otras es que una vez establecidos en destino, decidió separase de su pareja. Entonces,

\footnotetext{
17 Comunicación con Juana, Apolinario Saravia, septiembre de 2015.

${ }^{18}$ Ciudad Capital de la Provincia de Buenos Aires.
} 
rompió con el sistema de género de origen, dentro del cual no es habitual que las mujeres se separen. Esta capacidad de cuestionamiento del mandato de género fue excepcional y estuvo vinculado a la presencia de violencia física que sufría por parte del marido.

Entonces, nuevamente aparece la violencia machista como un acto de poder sobre el cuerpo de la mujer. Esa violencia física que sigue las leyes del patriarcado: la norma del control o posesión sobre el cuerpo femenino y la norma de la superioridad masculina (Segato, 2003). Esa violencia que tiene un papel fundamental en la reproducción del orden del género. En el caso de Natalia, la presencia de sus familiares en destino fue importante porque actuaron como un marco de contención que le permitió continuar trabajando (con su padre) en la horticultura y también recibir ayuda en el cuidado de sus hijos.

Entonces, al separarse, Natalia se transformó en jefa del hogar, única sostén del mismo a la vez que debía cuidar de sus hijos pequeños. Dentro de las estrategias que adoptó para realizar el doble trabajo productivo y reproductivo en ausencia del varón, reconoció la presencia de familiares colaborando en el cuidado de sus niños: "tenía el apoyo de mis hermanas, de mis hermanos también que están allá. Porque por ahí a veces me salvaba que los chicos se levanten de dormir y me los lleven. Entonces bueno decían, dejalos a las diez, once, a la hora que se levantan, vení a llevarlos. Ellos me los miraban"19.

Estos favores funcionan a través de la generación de obligaciones reciprocidad- que ella practica cuando cuida de su sobrina, una niña de un año, hija de su hermano. Justamente, al momento de la entrevista, Natalia se encontraba cocinando y cuidando de la niña. Situación que la colocaba como reproductora del rol femenino de cuidadora. De ese modo, a pesar de haber roto con ciertos mandatos femeninos cuando se separa, reproduce aquel rol tradicional de cuidadora y encargada de las tareas domésticas, que aparecen como incuestionables. Luego de varios años volvió a unirse en pareja con un varón boliviano y desde entonces trabaja junto a él en la horticultura.

El caso de María (mencionado anteriormente) también resulta interesante porque a partir del fallecimiento de su marido, se encargó de criar a sus hijas y sostener económicamente su hogar. Pasó a tener un rol preponderante en su unidad doméstica tomando las decisiones que atañen a la vida cotidiana de sus integrantes. Comenzó a organizar las tareas dentro del hogar y a administrar el dinero que ingresaba. También a realizar los arreglos contractuales con el patrón. De algún modo este hecho traumático la obligó a adoptar un rol que podría asociarse a lo masculino.

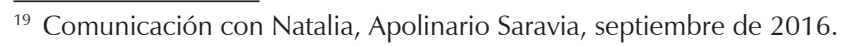


En una visita a la finca donde María se encontraba trabajando, hablamos con el patrón quien nos dijo "pero si es guapa ella, trabaja más que los varones" ${ }^{\prime 20}$. Para los patrones la pertenencia nacional y la condición de género: ser mujer y boliviana la convierte en buena trabajadora, sacrificada, aún por sobre los varones bolivianos. Esto podría pensarse a priori como algo que posiciona a las mujeres en mejores condiciones para negociar su situación laboral. Pero esto no es así, aquello que posicionaba a María como "buena trabajadora" también la asignaba a un lugar subordinado con respecto al patrón y de dependencia/subordinación. Como vimos, las mujeres aparecen como subalternas tanto en el orden familiar como laboral. Esta jerarquización laboral y familiar es resultado de su condición de género, etnia y de clase. Justamente, los patrones suelen aprovechar la imagen positiva etnicizada y generizada de "buena trabajadora" en el caso de las mujeres bolivianas, para reforzar prácticas explotadoras y, quizás de forma más intensa cuando ambos, patrón y trabajadora son de una misma etnicidad nacionalidad (Moore, 2011).

María nos contaba que, desde que falleció su marido depende de que algún hermano pueda y quiera venir a trabajar con ella. Cuando le preguntamos qué haría en caso de no contar con algún varón para trabajar, nos dijo: "tendré que trabajar como mensualera"21. Es decir tendría que, obligadamente, insertarse bajo una forma que se percibe como más precaria pues "pagan poco y hay que hacer lo que te pidan, a cualquier hora". Para María varias situaciones se conjugan en sus limitadas opciones laborales: su condición de género, sin una pareja o un varón con quien pueda asociarse para trabajar, pero también su condición de clase ya que no cuenta con tierras en origen para retornar, ni un capital para iniciar un negocio, que según ella son las únicas opciones viables para una mujer en su condición.

Ahora bien, más allá de la reproducción de las desigualdades reconocidas a lo largo de la investigación, encontramos ciertos factores que permitieron mejorar las condiciones de vida de las mujeres, fundamentalmente en aquellas identificadas como jefas de hogar. Es decir, quienes luego de separarse o de enviudar se convirtieron en único sostén económico del hogar. Algunas entrevistadas mencionaron la importancia de ciertos ingresos extra procedentes de políticas sociales, implementadas desde el Gobierno Nacional a finales de la década del 2000, que aparecieron como sustanciales para sostener la economía familiar, cuya base es el ingreso procedente del trabajo en las hortalizas. Entre aquellos ingresos, mencionaron principalmente la Asignación Universal por Hijo, pero también la Tarjeta social.

\footnotetext{
20 Nota del cuaderno de campo, mayo de 2016.

21 Comunicación con María, Apolinario Saravia, septiembre de 2015.
} 


\section{Conclusiones}

A partir de los casos analizados, hemos observado que los varones iniciaron la migración previo a que lo hicieran las mujeres. Estos varones migraron siguiendo el mandato de proveedores o de valerse por sí mismos. En cambio las mujeres migraron generalmente por reunificación familiar, guiadas por el objetivo de constituir su unidad doméstica como ámbito de reproducción de la maternidad. En muy pocos casos observamos migraciones autónomas, y en esos casos, las mujeres migraron con varones que integraban su familia nuclear o ampliada y una vez en destino, conformaron parejas con varones de sus lugares de origen. No obstante, más allá de estos casos excepcionales, dentro del sistema de género de origen, la forma legítima de migrar para las mujeres es con un varón con quien mantengan una relación de pareja. Y justamente, en pareja se asociaron para trabajar en destino, en la producción hortícola generalmente bajo la figura de la mediería.

En definitiva, lo que observamos es que el ámbito donde se negocia la migración es la familia, la unidad doméstica, la cual como planteamos está atravesada por relaciones de poder desigual y configurada por la ideología patriarcal. A su vez, las mujeres migran con sus parejas, a través de las redes masculinas que esos varones construyeron muchas veces en su experiencia migratoria previa. Esto las condiciona en su accionar, aunque como vimos, ellas logran romper con ciertos mandatos (por ejemplo se separan) cuando presentan redes de contención en destino como familiares o bien amigas/ comadres. Podemos inferir que las posibilidades de negociar mayores niveles de libertad o mostrar resistencia frente a algún tipo de situación de opresión, en los primeros tiempos de llegada, son ciertamente escasas. En este sentido la antigüedad de la migración repercute en la posibilidad de cuestionar el poder masculino.

Inclusive, pudimos visualizar ciertas condiciones de contexto, en determinados momentos de la experiencia migratoria, que permitieron contrarrestar las situaciones de opresión y favorecer procesos de mayor autonomía de las mujeres. En especial en aquellas que se convirtieron en único sostén económico de sus hogares, luego de una separación o enviudar. Nos referimos a la aparición de ciertas políticas públicas en destino. Podemos mencionar la implementación a partir del año 2009 de la Tarjeta Social y fundamentalmente de la Asignación Universal por Hijo que son identificadas por las mismas mujeres como importantes ingresos económicos complementarios.

Ahora bien, esas mujeres se insertan conjuntamente con sus parejas en un nicho laboral segmentado no sólo por etnia nacionalidad boliviana sino también por género, donde las decisiones son tomadas mayormente por los varones. Esto significa que los arreglos contractuales laborales, comerciales 
o de asesoramiento técnico, suelen realizarse entre varones. De esa forma, las mujeres quedan de algún modo atrapadas en las jerarquías masculinas, entrelazadas en la esfera laboral y doméstica y, en consecuencia son más vulnerables que ellos.

Es decir, si bien tanto varones como mujeres, se insertan en una actividad caracterizada por la informalidad, precariedad e inseguridad como es la actividad hortícola. En los varones la subordinación se encuentra atravesada por su condición de clase y de nacionalidad. En cambio, en las mujeres la subordinación está atravesada por su condición de clase, por su nacionalidad y se profundiza por su condición de género. Como dijimos las mujeres bolivianas se ubican en una posición subordinada como trabajadoras, con respecto a sus patrones, pero también dentro de la unidad doméstica, en relación a sus parejas.

En ese sentido, es posible afirmar, como lo hace Tapia Ladino (2011, p. 365)

... que las relaciones de género que predominaban antes de la partida no cambiaron de manera sustantiva con la migración. Las identidades, normas e ideologías de género predominantes en la sociedad boliviana que establecen una situación de subordinación de las mujeres y propician la mantención de la jerarquía patriarcal, no son revertidas por el mero hecho de cruzar las fronteras.

Y esto está vinculado principalmente al hecho de que el sistema de género en destino y en particular en la producción hortícola, responde a la misma lógica patriarcal que en origen.

\section{Bibliografía}

ARIZA, Marina. Itinerarios de los estudios de género y migración en México. In: ARIZA, Marina; PORTES Alejandro (coords.). El país transnacional: migración mexicana y cambio social a través de la frontera. México: UNAM, Instituto de Investigaciones Sociales, 2007, p. 453-515.

ATAIDE, Soraya. Género y trayectorias migratorias. El caso de tarijeños y tarijeñas vinculados al mercado de trabajo hortícola de Apolinario Saravia, provincia de Salta (Argentina) (Tesis de Doctorado en Ciencias Sociales). Ciudad de Buenos Aires: Facultad de Ciencias Sociales, Universidad de Buenos Aires, 2017.

BASTIA, Tanja. La reproducción de las desigualdades de género en origen y en destino: un estudio transnacional a partir de las migraciones bolivianas. Papeles del CEIC, v. 2014/2, n. 110, Centro de Estudios sobre la Identidad Colectiva, Universidad del País Vasco, 2014.

BENENCIA, Roberto. Participación de los inmigrantes bolivianos en espacios específicos de la producción hortícola en la Argentina. Política y Sociedad, v. 49, n. 1, p. 163-178, 2012. 
CASSANELLO, Carina Alejandra. Historia reciente de los inmigrantes bolivianos en la Argentina, 1970-2000. Trayectorias migrantes, redes sociales y transnacionalidad (Tesis de posgrado). Bernal, Argentina: Universidad Nacional de Quilmes, 2014.

COURTIS, Corina; PACCECA, María Inés. Género y trayectoria migratoria: mujeres migrantes y trabajo doméstico en el Área Metropolitana de Buenos Aires. Papeles de Población, v. 16, n. 63, p. 155-185, enero-marzo 2010.

D'AUBETERRE BUZNEGO, María Eugenia. Cautivas en el laberinto: migración femenina a california, comercio informal e inserción en lo global. In: GÓMEZ, Martha Judith Sánchez; SERRA YOLDI, Inmaculada (coords.). Ellas se van: Mujeres migrantes en Estados Unidos y España. México: Instituto de Investigaciones Sociales, UNAM, 2013, p. 453-484.

DE BARBIERI, Teresa. Sobre la categoría de género: una introducción teóricometodológica. Fin de Siglo. Género y cambio civilizatorio. Chile: Ediciones de las Mujeres, n. 17, 1992.

GARCÍA, Matías. Análisis de las transformaciones de la estructura agraria hortícola platense en los últimos 20 años: El rol de los horticultores bolivianos (Tesis de Doctorado). Facultad de Ciencias Agrarias y Forestales Universidad Nacional de La Plata, 2012.

GIARRACA, Norma. Territorios y lugares. Entre las fincas y la ciudad, Lules en Tucumán. Buenos Aires: Editorial La Colmena, 2003.

GREGORIO GIL, Carmen. Migración Femenina. Su impacto en las relaciones de género. Madrid: Ediciones Narcea, 1998.

HERRERA, Gioconda. Género y migración internacional en la experiencia latinoamericana. De la visibilización del campo a una presencia selectiva. Política y Sociedad, v. 49, n. 1, p. 35-46, 2012.

HONDAGNEU-SOTELO, Pierrette. Gender and Migration Scholarship: An Overview from a 21st Century Perspective. Migraciones Internacionales, v. 6, n. 1, p. 219-233, 2011.

MAGLIANO, María José. Migración, género y desigualdad social. La migración de mujeres bolivianas hacia Argentina. Estudios feministas, Florianópolis, v. 17, n. 2, p. 349-367, mayo-agosto 2009.

MAHLER, Sarah; PESSAR, Patricia R. Gender Matters: Ethnographers Bring Gender from the Periphery toward the Core of Migration Studies. IMR, v. 40, n. 1, p. 28-63, Spring 2006.

MALLIMACCI BARRAL, Ana. Revisitando la relación entre géneros y migraciones. Resultados de una investigación en Argentina. Mora, v. 18, n. 2, Ciudad Autónoma de Buenos Aires, dic. 2012.

MOORE, Susana. La construcción social del trabajo de mujeres bolivianas en verdulerías de Buenos Aires. Trabajo, género y etnicidad-nacionalidad (Tesis para optar por el título de Magíster en Antropología Social). Facultad Latinoamericana de Ciencias Sociales, Sede Argentina, 2011. 
PEDONE, Claudia. 'Varones aventureros' vs. 'Madres que abandonan': reconstrucción de las relaciones familiares a partir de la migración ecuatoriana. REMHU, Revista Interdisciplinar da Mobilidade Humana, v. 16, n. 30, p. 45-64, 2008.

PIZARRO Cynthia Alejandra. Partir y volver entre Argentina y Bolivia: trayectorias migratorias transnacionales e intersección de desigualdades. Ponencia presentada en las VII Jornadas Santiago Wallace de Investigación en Antropología Social, Sección de Antropología Social, Instituto de Ciencias Antropológicas, Facultad de Filosofía y Letras, UBA, Buenos Aires, 2013.

PIZARRO, Cynthia. Bolivianos y bolivianas en la vida cotidiana cordobesa: Trabajo, derechos e identidad en contextos migratorios. Córdoba: Editorial de la Universidad Católica de Córdoba, 2015.

PIZARRO, Cynthia. Clasificar a los otros migrantes: las políticas migratorias argentinas como productoras de etnicidad y de desigualdad. MÉTIS: história \& cultura, v. 11, n. 22, p. 219-240, 2012.

PIZARRO, Cynthia. Sufriendo y resistiendo la segregación laboral: experiencias de migrantes bolivianos que trabajan en el sector hortícola de la región metropolitana de la ciudad de Córdoba. In: PIZARRO, Cynthia (coord.) Migraciones internacionales contemporáneas: estudios para el debate. CICCUS, 2011.

ROSAS, Carolina. Las participaciones migratorias de los varones y las concesiones del género. Veracruzanos (Mexicanos) en Chicago y peruanos en Buenos Aires. Revista Científica de UCES, v. XVII, n. 1, p. 111-131, Otoño 2013.

SEGATO, Rita. Las estructuras elementales de la violencia. Bernal: Universidad Nacional de Quilmes, 2003.

STEFONI, Carolina. Inmigración y ciudadanía: la formación de comunidades peruanas en Santiago y la emergencia de nuevos ciudadanos. Política, n. 43, p. 319-336, 2004.

TAPIA LADINO, Marcela. Las relaciones de pareja entre migrantes bolivianos/ as en Madrid: ¿cambios, resistencias o continuidades?. AlBR, Revista de Antropología Iberoamericana, v. 6, n. 3, p. 341-371, sep.-dic. 2011. 